\title{
Elucidating the Role of Viral Non-structural Proteins on the Morphogenesis of Arterivirus Replication Structures
}

\author{
Barbara van der Hoeven ${ }^{1}$, Eric J. Snijder ${ }^{2}$, Kèvin Knoops ${ }^{1,3}$, Abraham J. Koster ${ }^{1}$, Montserrat \\ Bárcena ${ }^{1}$ \\ 1. Dept. of Molecular Cell Biology, Section Electron Microscopy, Leiden University Medical \\ Center, The Netherlands. \\ 2. Dept. of Medical Microbiology, Molecular Virology Laboratory, Leiden University Medical \\ Center, Leiden, The Netherlands. \\ 3. Dept. of Molecular Cell Biology, Groningen Biomolecular and Biotechnical Institute, \\ Groningen, The Netherlands.
}

Positive-strand RNA (+RNA) viruses comprise the largest group of viruses and contain several human pathogens such as poliovirus, hepatitis $C$ virus and SARS-coronavirus (SARS-CoV). One characteristic of +RNA viruses is that their replication takes place in the cytoplasm. An essential element in this replication is the transformation of cellular membranes into specialized structures that support the synthesis of new viral RNA, also termed replication structures.

The process of appropriating host cellular membranes is driven by viral non-structural proteins (nsps), several of which are predicted to interact with host membranes. For equine arteritis virus (EAV), a member of the order Nidovirales, nsp2, nsp3 and nsp5 contain predicted transmembrane domains and are therefore candidates to be directly responsible for the formation of the replication structures. Indeed, it was shown that the co-expression of nsp2 and nsp3 is not only essential for the formation of these structures, but also sufficient to induce structures that resembled those found in infected cells $[1,2]$. These EAV-induced membrane modifications had been characterized at the time as double-membrane vesicles (DMVs) [3]. However, electron tomography (ET) in combination with improved ultrastructural preservation methods revealed that these membrane structures are not isolated DMVs, but are in fact part of a larger network comprising interconnected DMVs that are continuous with the endoplasmatic reticulum (ER) [4], an architecture similar to that found for the distantly-related nidovirus SARS-CoV [5]. This raises the question of whether nsp2 and 3 are capable of generating membrane modifications with this complex architecture.

In order to answer this question we used an expression system to induce the production of a GFPtagged nsp2-3 polyprotein that cleaves itself to form the active proteins. The fluorescence data shows that in the nsp2-3-GFP expression system nsp2 distribution (figure 1A) is similar to the perinuclear pattern typically seen in infected cells [6]. High-pressure freezing and freezesubstitution was used in order to retain excellent ultrastructural preservation. We used ET for the three-dimensional imaging of the nsp2-3 induced membrane modifications. Our preliminary 3D data confirms the ability of these two nsps to induce DMVs but, strikingly, also reveals structures that resemble possible intermediate structures. So far little is known about the origin of these membrane modifications as intermediate structures are never detected in wild-type infection, presumably as these are rapid and transient stages. Two mechanisms have been proposed for the formation of DMVs from ER: enwrapping and double-budding [3]. The results so far seem to favor the enwrapping mechanism as openings of the DMV to the cytosol are 
observed (figure1B), which are predicted in the corresponding mechanism to be intermediate structures.

Additionally, these preliminary results also show that there are differences between wild-type structures and those induced by expressing nsp2-3. This strongly suggests that other viral proteins or components are necessary for full maturation of replication structures. Experiments using added viral proteins, particularly nsp5 which is the third protein predicted to have a transmembrane region, will shed light on this aspect.

Our results indicate that this system might offer a unique look into the morphogenesis of these structures as this seems to halt the formation of DMVs during their formation. Further analysis will enable us to determine by which mechanism DMVs are formed and to clarify the function of the viral nsps involved.

\section{References:}

[1] EJ Snijder et al, Journal of General Virology 82 (2001), p. 985.

[2] CC Posthuma, et al, Journal of Virology 82 (2008), p. 4480.

[3] KW Pedersen et al, Journal of Virology 73 (1999), p. 2016.

[4] K Knoops et al, PLoS Biology 6 (2008), p. e226.

[5] K Knoops et al, Journal of Virology 86 (2012), p. 2474.

[6] Y van der Meer et al, The Journal of Virology 72 (1998), p. 6689.

[7] The authors acknowledge funding from NWO, grants CW-TOP 700.57.301 and MEERVOUD-836.10.003.
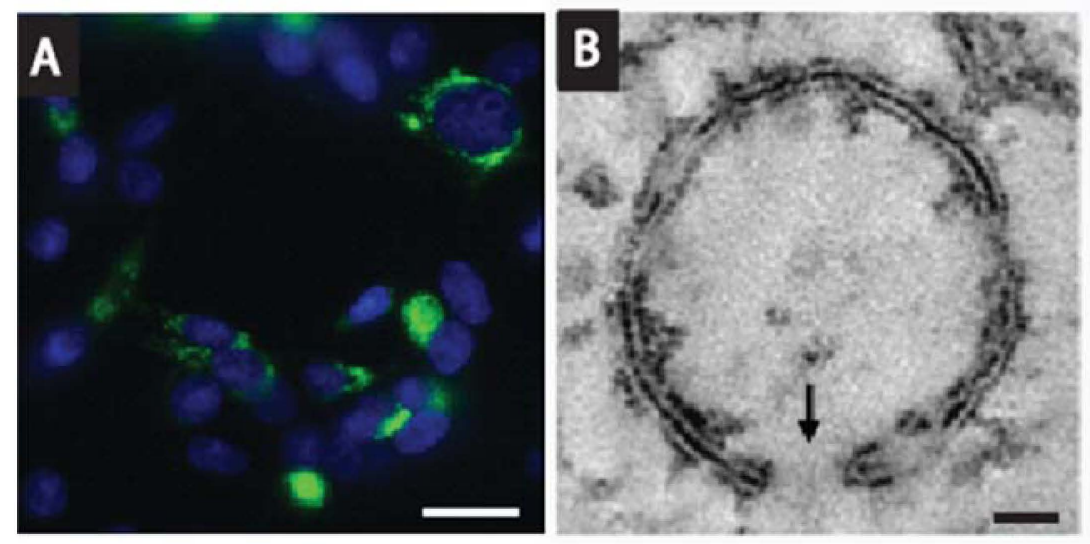

Figure 1. Structures induced by nsp2-3 A: Fluorescence image showing cells expressing the nsp2-3 GFP construct with additional Hoechst nuclear staining. Bar, $20 \mu \mathrm{m}$.

B: Virtual slice through an electron tomogram of one of the nsp2-3 induced structures. In contrast with wild-type DMVs this vesicle is open to the cytosol (arrow) Bar 100nm. 